\title{
Zero-Flow Pressures and Pressure-Flow Relationships during Single Long Diastoles in the Canine Coronary Bed before and during Maximum Vasodilation
}

\author{
LIMITED INFLUENCE OF CAPACITIVE EFFECTS
}

\author{
Francis J. Klocke, Irwin R. Weinstein, James F. Klocke, Avery K. Ellis, \\ David R. Kraus, Robert E. Mates, John M. Canty, Ran D. ANbar, \\ Roslyn R. Romanowski, KenNeth W. Wallmeyer, and Martin P. Echt, \\ Departments of Medicine, Mechanical Engineering, and Physiology, State \\ University of New York at Buffalo, Erie County Medical Center, \\ Buffalo, New York 14214
}

\begin{abstract}
A в S T RACT The proposal that diastolic coronary flow is regulated by an intramyocardial "back-pressure" that substantially exceeds coronary venous and ventricular diastolic pressures has been examined in an open-chest canine preparation in which instantaneous left circumflex pressure and flow could be followed to cessation of inflow during prolonged diastoles. Despite correlation coefficients consistently $>0.90$, pressureflow data during individual diastoles were concave to the flow axis before and during pharmacologically induced maximum coronary vasodilation. Data were better fitted $(P<0.01)$ by second-order equations than by linear equations in $>90 \%$ of cases. Second-order pressure-axis intercepts $\left(\mathrm{P}_{\mathrm{f}=0}\right)^{1}$ averaged $29 \pm 7$ (SD) $\mathrm{mm} \mathrm{Hg}$ before vasodilation and $15 \pm 2 \mathrm{~mm} \mathrm{Hg}$ during vasodilation; left and right atrial pressures were always substantially lower $(8 \pm 3$ and $5 \pm 2 \mathrm{~mm} \mathrm{Hg}$ before vasodilation and $8 \pm 2$ and $4 \pm 1 \mathrm{~mm} \mathrm{Hg}$ during dilation). Values of $P_{f=0}$ before vasodilation varied directly with levels of coronary inflow pressure. A modification of the experimental preparation in which diastolic circumflex pressure could be kept constant was used to evaluate the suggestion that $P_{f=0}$ measured during long diastoles are misleadingly high because of capacitive effects within the coronary circulation as inflow pressure decreases. Decreases in $P_{f=0}$ attributable
\end{abstract}

Address all correspondence to Dr. Klocke, Erie County Medical Center.

Received for publication 17 March 1981 and in revised form 8 June 1981.

${ }^{1}$ Abbreviations used in this paper: LC, left circumflex; $\mathbf{P}_{\mathrm{f}=0 \text {, }}$, zero-flow pressure-axis intercept. to capacitive effects averaged only $5.9 \pm 3.0 \mathrm{~mm} \mathrm{Hg}$ before vasodilation and were smaller during dilation. We conclude that $P_{f=0}$ is a quantitatively important determinant of coronary driving pressure and flow, resulting from both factors related to, and independent of, vasomotor tone. Adjustments of flow during changing physiological situations may involve significant changes in $\mathrm{P}_{\mathrm{f}=0}$ as well as in coronary resistance.

\section{INTRODUCTION}

Traditional views of coronary driving pressure and resistance have been called into question by Bellamy's retrospective analysis of records of instantaneous coronary pressure and left circumflex inflow during single diastoles in chronically instrumented dogs with low resting heart rates (1). Under semibasal conditions the relationship between diastolic inflow and coronary inflow pressure was felt to be linear over the range of pressure studied; extrapolation of the pressure-flow line gave a zero-flow pressure-axis intercept $\left(\mathrm{P}_{\mathrm{f}=0}\right)$ of 40-50 mm $\mathrm{Hg}$. The latter finding suggests a "backpressure" to flow of hitherto unsuspected magnitude in the normal coronary bed, and implies that coronary driving pressure-considered as the difference between inflow pressure and the minimum pressure required for forward flow during diastole - has been overestimated seriously in previous studies. Additional observations in four animals during reactive hyperemia, and in a single animal during adenosine infusion, indicated that $P_{\mathrm{f}=0}$ can fall at least to $20 \mathrm{~mm} \mathrm{Hg}$ in response to vasodilatory stimuli. Since most diastoles were $<2 \mathrm{~s}$ in length during these latter studies, the degree of extrapolation required at high flows to obtain 
$P_{f=0}$ was larger than under basal conditions. Systematic studies of $P_{\mathrm{f}=0}$ during steady-state maximum coronary vasodilation were not reported.

Since the relationship between circumflex inflow and pressure represents a summation of relationships in multiple downstream parallel channels, the linearity of the relationship observed by Bellamy is in some respects surprising. Transmural differences in zeroflow pressure have been suggested by two recent studies $(2,3)$ and would be expected to produce curvilinearity of the pressure-flow relationship as inflow approaches zero. If the value of $P_{f=0}$ obtained from the measurement of total inflow represents the lowest detectible $P_{f=0}$ among a family of $P_{f=0}$, overall conductance should decrease as vascular channels with higher $P_{f=0}$ drop out of the inflow measurement. Autoregulatory changes in resistance, passive changes in distending pressure and/or intravascular volume, changes in apparent viscosity at low flow rates, and other factors might also affect the overall pressure-flow relationship during a long diastole.

These and other potential limitations in the interpretation of Bellamy's provocative data have been carefully pointed out by Bellamy $(1,4,5)$, and others (6). Because of the conceptual implications of values of $P_{f=0}$ that exceed coronary outflow pressure and/or intraventricular diastolic pressure, interest has focused on factors that may cause measurements of $P_{f=0}$ to be misleadingly high, or to differ in different experimental settings. The present studies were intended to address these issues by defining diastolic circumflex pressureflow relationships in settings in which diastolic inflow could be followed to zero during a single cardiac cycle, both with autoregulation intact and during sustained maximum vasodilation. Values of $P_{f=0}$ were compared with simultaneous values of right and left atrial pressure, the former reflecting coronary venous outflow pressure and the latter the commonly presumed maximum value of diastolic intramyocardial tissue pressure. Linear and nonlinear fitting procedures were compared for each pressure-flow relationship, and reproducibility of $\mathrm{P}_{\mathrm{f}=0}$ was tested under standardized hemodynamic conditions. Pressure-flow relationships were also obtained in individual animals at different inflow pressures to evaluate the possibility that changes in $P_{f=0}$ play a role in adjustments of coronary flow which have traditionally been attributed predominantly to changes in resistance. Finally, a modification of the basic experimental preparation in which diastolic circumflex pressure could be kept constant was used to evaluate the recent suggestion of Eng et al. (7) that measurements of circumflex inflow importantly underestimate flow at the capillary level during a long diastole because of capacitive discharge of blood from precapillary vessels as inflow pressure falls.

\section{METHODS}

The experimental preparation is outlined in Fig. 1. Mongrel dogs anesthetized with pentobarbital underwent left thoracotomy and had pacing electrodes sewn onto the right ventricle. Heart block was produced by formaldehyde injection into the atrioventricular node (8) following bilateral vagotomy, and the ventricles paced at 100 beats $/ \mathrm{min}$. Mean and phasic left circumflex (LC) arterial inflow were measured continuously. In $\sim 60 \%$ of the animals studied, the LC was cannulated directly and perfused through a circuit of rigid tubing originating in the left subclavian artery and incorporating an in-line electromagnetic flow probe and short segment of tubing, which could be occluded for recording flowmeter zero. The flow probe was used in conjunction with a sine-wave flowmeter (model BL-613, Biotronex Laboratory, Kensington, Md.). In the remaining animals (upper right inset in Fig. 1), measurements were made with a cuff-type electromagnetic flow probe and the same sine-wave flowmeter; a cuff-type pneumatic occluder was positioned downstream to the probe for determination of flowmeter zero. Each probe was carefully fitted to the LC, with zero base-line levels required to be stable $< \pm 3 \mathrm{ml} / \mathrm{min}$ on repeated inflation of the occluder. In all animals downstream LC pressure was measured directly through a cannula inserted into a small epicardial branch of the LC or the distal LC itself.

A large-bore tube was positioned in the aorta and connected to a Y-tube. One arm of the tube led to a reservoir kept at a preselected height so that fluctuations in systolic pressure during the course of an experiment were minimized. The second arm was ordinarily clamped but could be vented to the atmosphere during long diastoles (at a rate controlled by a screw clamp), as outlined below. Thoracic aortic, left atrial, and left ventricular pressures were measured in all animals, the latter with a Konigsberg transducer. In $50 \%$ of animals right atrial pressure was also monitored. Hemoglobin concentrations ranged from 12 to 19 g/100 ml but varied by no more than $15 \%$ during studies in any individual animal. $\mathrm{O}_{2}$ was added to the inflow of the Harvard positive-pressure respirator to keep arterial $\mathrm{O}_{2}$ saturation at $\geq 95 \%$. Arterial $\mathrm{pH}, \mathrm{PCO}_{2}$, and $\mathrm{PO}_{2}$ averaged $7.35 \pm 0.03$ (SD), $36 \pm 4 \mathrm{~mm} \mathrm{Hg}$, and $153 \pm 44 \mathrm{~mm} \mathrm{Hg}$, respectively.

Because preliminary studies indicated that values of $P_{f=0}$ were sensitive to alterations in systemic pressure, initial studies were confined to animals stabilizing at a systolic pressure of $\geq 100 \mathrm{~mm} \mathrm{Hg}$ after the experimental preparation had been completed. At the time of each pressure-flow determination, the arm of the aortic Y-tube leading to the elevated reservoir was clamped and the pacemaker turned off at endexpiration. Because of normal postpacing suppression of spontaneous ventricular escape $(9,10)$, a diastole lasting 2$8 \mathrm{~s}$ ensued. In the majority of determinations, the second arm of the aortic Y-tube was vented to the atmosphere at the onset of the long diastole to obtain a sufficiently large decrease in LC pressure to ensure that LC inflow reached zero before ventricular escape. In most cases, ventricular escape was also delayed by administration of lidocaine. Values for all hemodynamic parameters, including instantaneous LC flow and pressure $\left(\mathrm{P}_{\mathrm{LC}}\right)$, were digitized at 10 -ms intervals throughout each long diastole and the six cardiac cycles immediately preceding it, and stored for later analysis. A mechanical flowmeter zero recording obtained during the first few seconds after each prolonged diastole was digitized similarly and used for the quantitation of flows during the long diastole and the six cardiac cycles immediately preceding it.

Prolonged diastoles were studied repeatedly at constant systemic pressure and a ventricular pacing rate of 100 in 27 animals. Nine of these animals were also studied during 

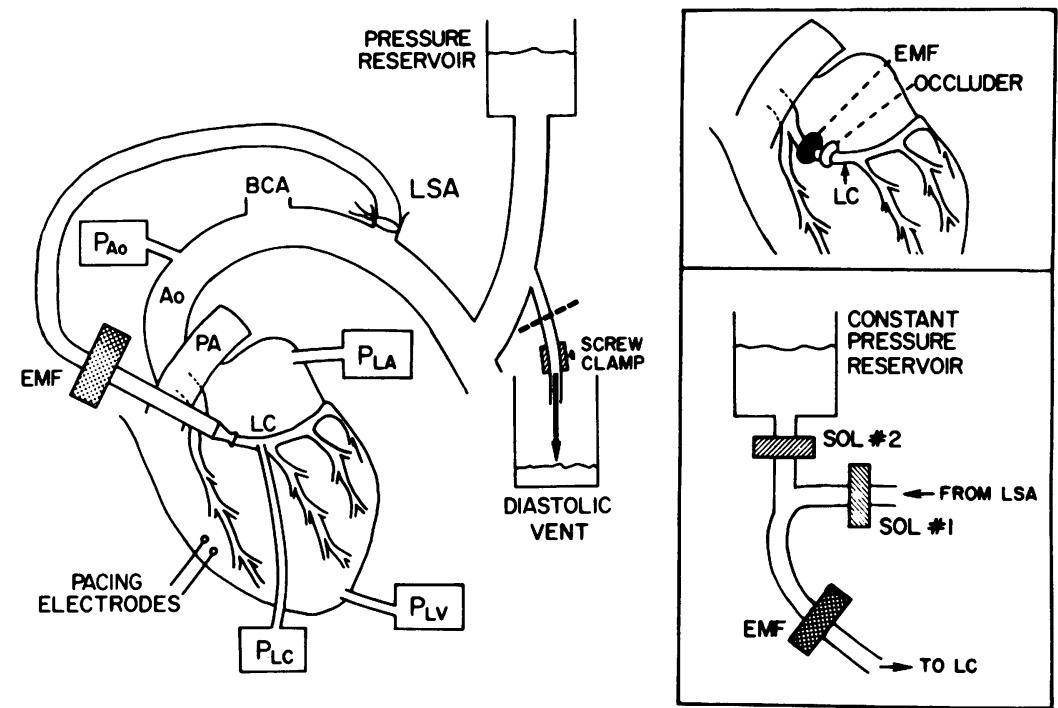

FIGURE 1 Experimental preparation. The basic preparation is shown on the left, with the LC perfused from the aorta through an external circuit incorporating an in-line electromagnetic flow probe (EMF). The inset on the upper right illustrates the use of a cuff-type probe applied directly to the circumflex artery. The inset on the lower right illustrates the modification of the basic preparation used to evaluate capacitive effects. See text for further details. Ao, aorta; PA, pulmonary artery; $\mathrm{BCA}$, brachiocephalic artery; LSA, left subclavian artery; $\mathbf{P}_{\mathrm{Ao}}, \mathbf{P}_{\mathrm{LA}}, \mathrm{P}_{\mathrm{LC}}$, and $P_{L V}$, aortic, left atrial, left circumflex, and left ventricular pressures, respectively; SOL = solenoid.

reductions in coronary inflow pressure. In 11 animals, studies were performed (at the animal's spontaneous aortic pressure) during maximum coronary vasodilation achieved by systemic administration of carbocromen, 5-8 mg/ $\mathrm{kg}$. Completeness of vasodilation was verified by the absence of reactive hyperemia after 30-60 s inflow occlusion.

Digitized values for LC pressure and flow were processed on a PDP-8 computer (Digital Equipment Corp., Maynard, Mass.) To minimize capacitive effects of refilling of vessels compressed during the previous systole, the onset of diastole was arbitrarily taken as $100 \mathrm{~ms}$ after left ventricular pressure fell to $40 \mathrm{~mm} \mathrm{Hg}$ during ventricular relaxation. Individual 10 -ms data points were smoothed by correcting each point to the average of itself and the two points immediately preceding and following. Points for further analysis were selected at pressure decrements of $0.5 \mathrm{~mm} \mathrm{Hg}$ (to avoid undue weighting of data collected during the later portions of diastole, when rates of change of pressure and flow were decreasing). These points were then subjected to linear- and second-order analysis with standard least squares curve-fitting techniques (and excluding data points occurring after LC inflow reached or passed through the zero-flow level). The equation forms used were $y=a X+b$ and $Y=a X^{2}$ $+b X+c$. The reduction in sum of squares associated with the second-order fit was tested against the mean-square remaining after second-order regression in an $f$ test, $P<0.01$ being taken as the level at which the second-order fit was justified (11).

The lower right inset in Fig. 1 illustrates the modification of the experimental preparation used to evaluate capacitive effects during long diastoles by comparing values of $P_{f=0}$ determined during variable- and constant-pressure LC perfusion. The LC was cannulated directly and, with paired solenoid valves, could be perfused from either the aorta (through the left subclavian artery) or a constant-pressure reservoir. Instantaneous LC inflow was again monitored with an in-line electromagnetic flow probe. LC pressure down- stream to the cannula was measured directly in the usual fashion. Before each experimental determination, the LC was perfused from the aorta in the usual fashion.

Pressure-flow relationships obtained from single postpacing diastoles during aortic perfusion were compared with the similar relationship defined by a series of points obtained by switching from aortic to reservoir perfusion at the onset of each of a series of long diastoles in which preselected reservoir pressures were varied. During diastoles with aortic perfusion (variable inflow pressure), the vent arm of the aortic Y-tube was again opened to the atmosphere to ensure that LC inflow reached zero. For postpacing diastoles with reservoir perfusion (constant inflow pressure), the solenoid in the coronary perfusion circuit connecting to the aorta was closed and the solenoid connecting to the constantpressure reservoir opened during the first 0.2 seconds of diastole. Although perfusion pressure remained constant throughout the long diastole, instantaneous values of LC inflow frequently varied (as described subsequently) and were followed until ventricular escape. Flowmeter zero was measured by occluding LC inflow after each long diastole, and again used for the quantitation of flows during the long diastole and the six normal cardiac cycles preceding it. Single-diastole, variable inflow pressure runs were interspersed with the several constant-pressure runs required to construct each constant-pressure relationship. Comparisons of variable- and constant-pressure relationships were attempted only when aortic systolic pressures immediately before each of the several runs required for the comparison varied by no more than $15 \mathrm{~mm} \mathrm{Hg}$.

\section{RESULTS}

Data from 27 animals studied at normal coronary inflow pressure before coronary vasodilation are presented in Table I and Figs. 2 and 3. Correlation 
TABLE I

Second-Order Values of $P_{\mathrm{f}=0}$ and Hemodynamic Data Immediately before Its Determination with Coronary Vasomotor Tone Intact*

\begin{tabular}{|c|c|c|c|c|c|c|}
\hline \multirow[b]{2}{*}{ Dog } & \multirow[b]{2}{*}{$n$} & \multicolumn{2}{|c|}{$\mathbf{P}_{\mathbf{A} 0}$} & \multirow[b]{2}{*}{$\mathbf{P}_{\mathrm{LA}}$} & \multirow[b]{2}{*}{$\mathbf{P}_{\mathbf{R A}}$} & \multirow{2}{*}{$\begin{array}{c}\text { Second-order } \\
\mathbf{P}_{t=0}\end{array}$} \\
\hline & & Systole & Diastole & & & \\
\hline & & \multicolumn{4}{|c|}{$m m \mathrm{Hg}$} & $m m \mathrm{Hg}$ \\
\hline $1 t$ & 6 & $115 \pm 2$ & $83 \pm 1$ & $6 \pm 0.4$ & $4 \pm 1$ & $26 \pm 3(21-31)$ \\
\hline $2 \ddagger$ & 6 & $103 \pm 5$ & $58 \pm 4$ & $6 \pm 3$ & $5 \pm 0.4$ & $18 \pm 4(14-23)$ \\
\hline $3 \ddagger$ & 6 & $109 \pm 3$ & $61 \pm 4$ & $6 \pm 1$ & $5 \pm 1$ & $20 \pm 3(17-25)$ \\
\hline $4 t$ & 6 & $100 \pm 5$ & $69 \pm 7$ & $5 \pm 1$ & $2 \pm 1$ & $22 \pm 3(18-25)$ \\
\hline $5 t$ & 4 & $107 \pm 3$ & $76 \pm 4$ & $6 \pm 0.1$ & $4 \pm 1$ & $22 \pm 1(21-24)$ \\
\hline $6 t$ & 12 & $118 \pm 5$ & $88 \pm 4$ & $7 \pm 1$ & $4 \pm 1$ & $29 \pm 4(22-33)$ \\
\hline 7 & 5 & $120 \pm 2$ & $73 \pm 3$ & $6 \pm 0.1$ & - & $33 \pm 2(29-34)$ \\
\hline $8 t$ & 3 & 117 & 85 & 9 & - & $45(40-47)$ \\
\hline 9 & 5 & $129 \pm 4$ & $91 \pm 2$ & $7 \pm 2$ & - & $30 \pm 5(25-36)$ \\
\hline 10 & 6 & $123 \pm 4$ & $93 \pm 2$ & $6 \pm 1$ & - & $24 \pm 3(21-29)$ \\
\hline 11 & 5 & $133 \pm 2$ & $80 \pm 6$ & $8 \pm 1$ & - & $33 \pm 2(29-35)$ \\
\hline 12 & 5 & $135 \pm 4$ & $95 \pm 4$ & $9 \pm 1$ & - & $30 \pm 1(28-32)$ \\
\hline 13 & 2 & 121 & 94 & 15 & - & $39 \quad(38-39)$ \\
\hline 14 & 6 & $121 \pm 8$ & $87 \pm 12$ & $10 \pm 1$ & - & $25 \pm 2(23-28)$ \\
\hline $15 \ddagger$ & 9 & $153 \pm 13$ & $114 \pm 12$ & $11 \pm 1$ & - & $34 \pm 3(31-39)$ \\
\hline 16 & 5 & $129 \pm 6$ & $94 \pm 10$ & $4 \pm 1$ & - & $40 \pm 2(38-42)$ \\
\hline 17 & 9 & $130 \pm 6$ & $95 \pm 8$ & $4 \pm 1$ & - & $24 \pm 1(22-26)$ \\
\hline 18 & 12 & $139 \pm 12$ & $107 \pm 9$ & $9 \pm 1$ & - & $35 \pm 3(30-39)$ \\
\hline 19 & 5 & $148 \pm 4$ & $82 \pm 4$ & $9 \pm 1$ & - & $26 \pm 2(23-28)$ \\
\hline 20 & 6 & $121 \pm 8$ & $88 \pm 10$ & $5 \pm 1$ & $5 \pm 1$ & $25 \pm 1(24-27)$ \\
\hline 21 & 3 & 107 & 67 & 18 & 9 & $22(21-24)$ \\
\hline 22 & 6 & $128 \pm 8$ & $81 \pm 6$ & $5 \pm 1$ & $2 \pm 0.4$ & $23 \pm 1(22-25)$ \\
\hline 23 & 8 & $124 \pm 5$ & $82 \pm 6$ & $14 \pm 1$ & $4 \pm 1$ & $30 \pm 3(27-34)$ \\
\hline 24 & 5 & $116 \pm 2$ & $87 \pm 5$ & $8 \pm 0.4$ & $6 \pm 0.2$ & $28 \pm 3(23-32)$ \\
\hline 25 & 5 & $127 \pm 14$ & $79 \pm 11$ & $8 \pm 1$ & $5 \pm 2$ & $37 \pm 3(34-40)$ \\
\hline 26 & 7 & $111 \pm 2$ & $82 \pm 5$ & $6 \pm 1$ & $5 \pm 1$ & $29 \pm 2(27-31)$ \\
\hline 27 & 6 & $108 \pm 8$ & $58 \pm 8$ & $12 \pm 2$ & $6 \pm 1$ & $24 \pm 2(20-26)$ \\
\hline Mean & & 122 & 83 & 8 & 5 & 29 \\
\hline SD & & 13 & 13 & 3 & 2 & 7 \\
\hline
\end{tabular}

$P_{f=0}$, zero-flow pressure. $P_{A o}, P_{L A}, P_{R A}$, aortic, left atrial, and right atrial pressures.

$*$ Values shown for each animal are mean $\pm \mathrm{SD} ; n=$ number of determinations in each animal. Values for $P_{t=0}$ in parentheses are the range of $P_{t=0}$ in individual animals. Times required for in flow to reach zero during long diastoles averaged $4.4 \pm 1.9 \mathrm{~s}$.

$\ddagger$ In these animals flow was measured with a cuff-type rather than an in-line electromagnetic probe.

coefficients for the total of 163 diastolic pressureflow relationships averaged $0.95 \pm 0.05$. Pressure-flow relationships, however, consistently showed concavity to the flow axis at lower inflow pressures and data were better fit $(P<0.01)$ by a second-order process than by a linear process in $91 \%$ of cases. Average values for $P_{f=0}$, i.e., pressure-axis intercepts, for second-order fits in all 27 animals were $29 \pm 7 \mathrm{~mm} \mathrm{Hg}$ (range 18-45 $\mathrm{mm} \mathrm{Hg}$ ). Mean left and right atrial pressures averaged $8 \pm 3$ and $5 \pm 2 \mathrm{~mm} \mathrm{Hg}$, respectively. Values for $P_{f=0}$ obtained from linear fits to all data points were slightly but systematically higher than with second-order fits, averaging $31 \pm 7 \mathrm{~mm} \mathrm{Hg}(P .<0.001$, paired $t$ test); individual differences between linear and second-order fits averaged $2.4 \pm 2.9 \mathrm{~mm} \mathrm{Hg}$. To evaluate effects of extrapolation to the pressure axis when inflow cannot be followed to zero, straight lines were also fitted to data points $>40 \mathrm{~mm} \mathrm{Hg}$. Values for $P_{f=0}$ were noticeably higher, averaging $35 \pm 6(P<0.001) \mathrm{mm} \mathrm{Hg}$; individual differences between these linear data fits and the original second-order fits averaged $6.8 \pm 4.3$ $\mathrm{mm} \mathrm{Hg}$.

The correlation coefficient between average secondorder values of $P_{f=0}$ and average values of mean $P_{L C}$ for the six cardiac cycles immediately preceding the long diastole used to determine $P_{\mathrm{f}=0}$ was 0.64 ( $n$ $=27, P<0.01$ ). Figs. 4 and 5 illustrate systematic reductions in $P_{f=0}$ with decreases in $P_{L C}$ in individual animals.

Data from the 11 animals studied during maximum 


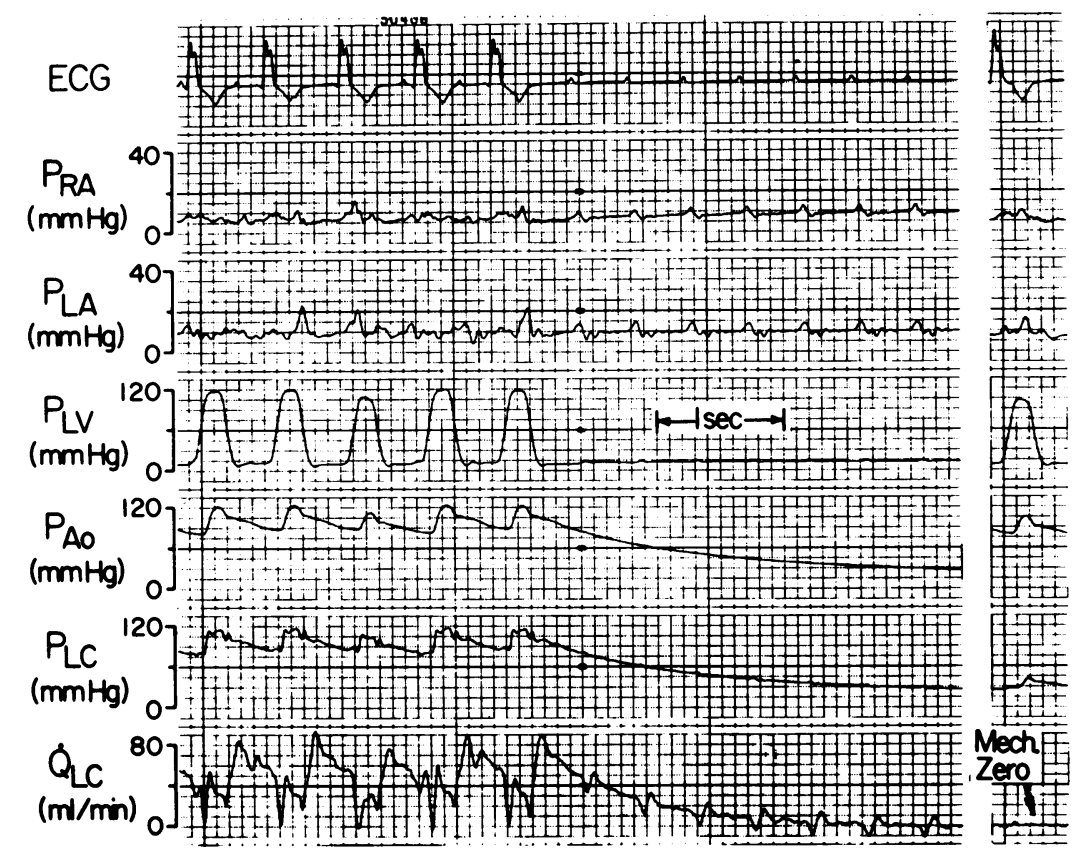

Figure 2 Analog record from a prolonged diastole (before vasodilation) in which LC inflow was measured with a cuff-type electromagnetic flow probe. $P_{R A}, P_{L A}, P_{L V}, P_{A 0}, P_{L C}$, pressures in the right atrium, left atrium, left ventricle, thoracic aorta, and left circumflex artery, respectively. $\dot{Q}_{\mathrm{LC}}$, left circumflex inflow; in this particular record, fluctuations in flow associated with atrial contraction (so-called "atrial coves") are apparent. Mech. zero, flowmeter zero recorded during brief mechanical occlusion of the circumflex perfusion circuit. Zero-flow pressure $\left(P_{f=0}\right)$ is $28 \mathrm{~mm} \mathrm{Hg}$.

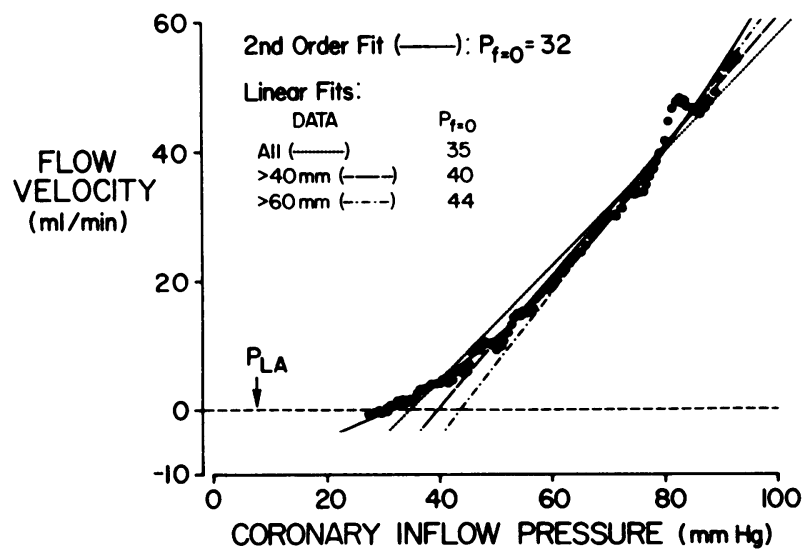

Figure 3 Pressure-flow relationships during a single long diastole before vasodilation. Individual data points are shown by solid circles. Although the correlation coefficient is 0.98 , the data show concavity to the flow axis at lower pressures and are better fit by a second-order equation than by a linear equation. Second-order zero-flow pressure $\left(P_{f=0}\right), 32 \mathrm{~mm}$ $\mathrm{Hg}$, substantially exceeds left atrial pressure $\left(\mathrm{P}_{\mathrm{LA}}\right), 7 \mathrm{~mm}$ $\mathrm{Hg}$. Right atrial pressure (not shown) was $5 \mathrm{~mm} \mathrm{Hg}$. Linear fits are shown for all data points, for data points $>40 \mathrm{~mm} \mathrm{Hg}$, and for data points $>60 \mathrm{~mm} \mathrm{Hg}$. In the latter two cases, $P_{f=0}$ derived by extrapolation is noticeably higher than that obtained from the second-order fit. vasodilation are presented in Table II and Figs. 6 and 7. Vasodilation was accompanied by four- to sixfold increments in flow at all pressure levels. Correlation coefficients for the total of 54 diastolic pressure-flow relationships averaged $0.96 \pm 0.06$. Pressure-flow relationships were again consistently concave to the flow

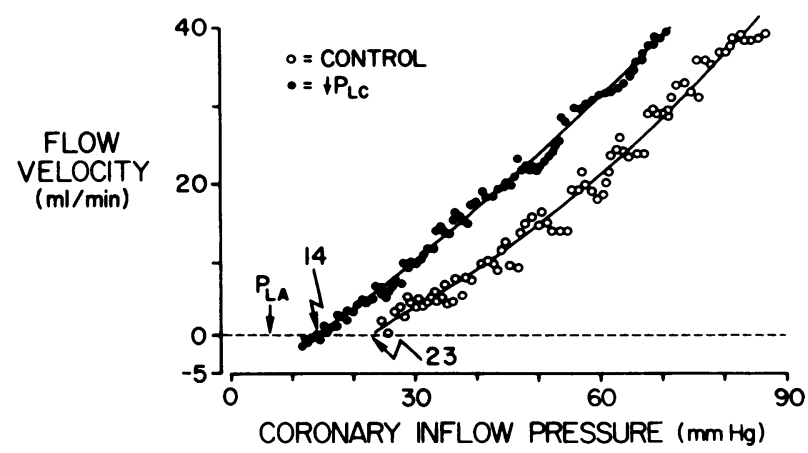

Figure 4 Pressure-flow relationships during a long diastole before and during modest reduction of circumflex pressure $\left(P_{L C}\right)$. The reduction was effected by constriction of the circumflex perfusion circuit. Zero-flow pressure $\left(P_{f=0}\right)$ decreases from 23 to $14 \mathrm{~mm} \mathrm{Hg}$ but remains above left atrial pressure $\left(P_{\mathrm{LA}}\right), 6 \mathrm{~mm} \mathrm{Hg}$. In this case, the reduction in $P_{f=0}$ was not associated with a discernible change in the shape (i.e., the slope) of the pressure-flow relationship. 


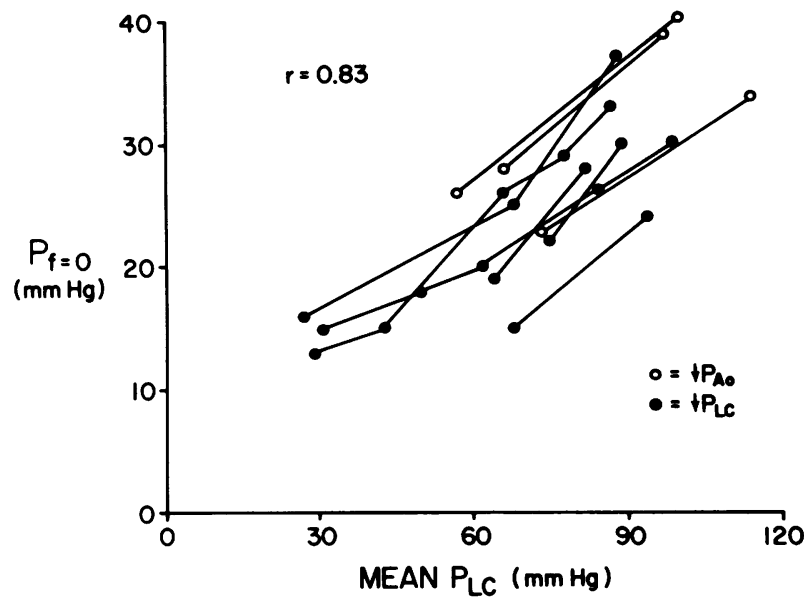

Figure 5 Relationship between zero-flow pressure $\left(P_{f=0}\right)$ and mean circumflex pressure $\left(\mathbf{P}_{\mathrm{LC}}\right)$ immediately before the determination of $P_{f=0}$ in nine animals studied at different levels of $P_{L C}$. In the three animals shown by open circles, $P_{L C}$ was reduced by temporary blood withdrawal from the aorta $\left(\downarrow P_{A 0}\right)$. In the six animals shown by solid circles, the reductions were achieved by constriction of the circumflex inflow circuit $\left(\downarrow P_{L C}\right)$. Each individual point represents the average of at least two pressure-flow determinations. The correlation coefficient for all 25 points is 0.83 .

axis and data were better fit $(P<0.01)$ by a secondorder than by a linear process in $93 \%$ of cases. Values for $P_{f=0}$ for second-order fits averaged $15 \pm 2 \mathrm{~mm} \mathrm{Hg}$ (range $13-18 \mathrm{~mm} \mathrm{Hg}$ ). Mean left and right atrial pres- sures were $8 \pm 2$ and $4 \pm 1 \mathrm{~mm} \mathrm{Hg}$, respectively. Values for $P_{f=0}$ obtained from linear fits for all data points were again slightly but systematically higher than with second-order fits, averaging $17 \pm 4 \mathrm{~mm} \mathrm{Hg}$ $(P<0.001$, paired $t$ test $)$; individual differences between linear and second-order fits averaged 2.8 \pm 3.1 $\mathrm{mm} \mathrm{Hg}$. Straight lines were also fitted to data points $>40 \mathrm{~mm} \mathrm{Hg}$; values for $\mathrm{P}_{\mathrm{f}=0}$ averaged $25 \pm 5 \mathrm{~mm} \mathrm{Hg}$ $(P<0.001)$, with individual differences between linear and original second-order fits averaging $11 \pm 5.6 \mathrm{~mm} \mathrm{Hg}$.

Representative pressure-flow data obtained in a single animal during long diastoles with constant-pressure reservoir perfusion (before vasodilation) are shown in Fig. 8. Even though LC pressure remained constant throughout each long diastole, LC inflow decreased with time when inflow pressure was $\geq \sim 60$ $\mathrm{mm} \mathrm{Hg}$. Flow variations with time were less at lower inflow pressures and had little effect on the pressureaxis intercept defined by the series of long diastoles.

Fig. 9 summarizes studies of capacitive effects in another animal, illustrating findings during variablepressure aortic perfusion as well as constant-pressure reservoir perfusion. Flows during constantpressure perfusion are slightly higher than flows at the same inflow pressure during aortic perfusion, but the reduction in $P_{f=0}$ for constant- vs. variable-perfusion pressure is only $4 \mathrm{~mm} \mathrm{Hg}$. Table III lists values for $P_{f=0}$ before vasodilation in 12 animals. $P_{f=0}$ during constant-pressure perfusion is slightly but systemati-

TABLE II

Second-Order Values of $P_{\mathrm{f}=0}$ and Hemodynamic Data Immediately before Its Determination during Maximum Coronary Vasodilation*

\begin{tabular}{|c|c|c|c|c|c|c|}
\hline \multirow[b]{2}{*}{ Dog } & \multirow[b]{2}{*}{$n$} & \multicolumn{2}{|c|}{$\mathbf{P}_{\mathbf{A} 0}$} & \multirow[b]{2}{*}{$P_{L A}$} & \multirow[b]{2}{*}{$\mathbf{P}_{\mathrm{RA}}$} & \multirow{2}{*}{$\begin{array}{c}\text { Second-order } \\
P_{t=0}\end{array}$} \\
\hline & & Systole & Diastole & & & \\
\hline & & \multicolumn{2}{|c|}{$m m \mathbf{H g}$} & \multicolumn{2}{|c|}{$m m \mathrm{Hg}$} & $m m \mathrm{Hg}$ \\
\hline 1t & 5 & $109 \pm 4$ & $77 \pm 2$ & $7 \pm 1$ & $5 \pm 1$ & $14 \pm 1(13-16)$ \\
\hline $2 \ddagger$ & 4 & $107 \pm 2$ & $57 \pm 3$ & $4 \pm 3$ & $6 \pm 1$ & $14 \pm 3(11-17)$ \\
\hline $6 \$$ & 11 & $120 \pm 6$ & $80 \pm 4$ & $8 \pm 1$ & $3 \pm 1$ & $13 \pm 2(10-16)$ \\
\hline $8 \ddagger$ & 2 & 125 & 88 & 8 & - & $17(17-17)$ \\
\hline 9 & 5 & $113 \pm 6$ & $68 \pm 7$ & $7 \pm 1$ & - & $17 \pm 1(16-18)$ \\
\hline 10 & 4 & $119 \pm 3$ & $78 \pm 2$ & $11 \pm 1$ & - & $16 \pm 1(15-17)$ \\
\hline 13 & 4 & $103 \pm 9$ & $54 \pm 7$ & $9 \pm 2$ & - & $18 \pm 2(16-20)$ \\
\hline 20 & 4 & $121 \pm 4$ & $76 \pm 1$ & $6 \pm 2$ & $5 \pm 0.1$ & $12 \pm 2(11-14)$ \\
\hline $22 \S$ & 3 & 103 & 53 & 10 & 4 & $15(14-17)$ \\
\hline $28 \S$ & 3 & 121 & 42 & 6 & 5 & $12(11-13)$ \\
\hline 29 & 3 & 108 & 48 & 10 & 3 & $13(13-14)$ \\
\hline Mean & & 114 & 66 & 8 & 4 & 15 \\
\hline SD & & 8 & 15 & 2 & 1 & 2 \\
\hline
\end{tabular}

* Format as in Table I. Times required for inflow to reach zero during long diastoles averaged $4.4 \pm 1.8 \mathrm{~s}$.

I In these animals flow was measured with a cuff-type rather than an in-line electromagnetic probe.

$\S \mathrm{HR}, 120$. 


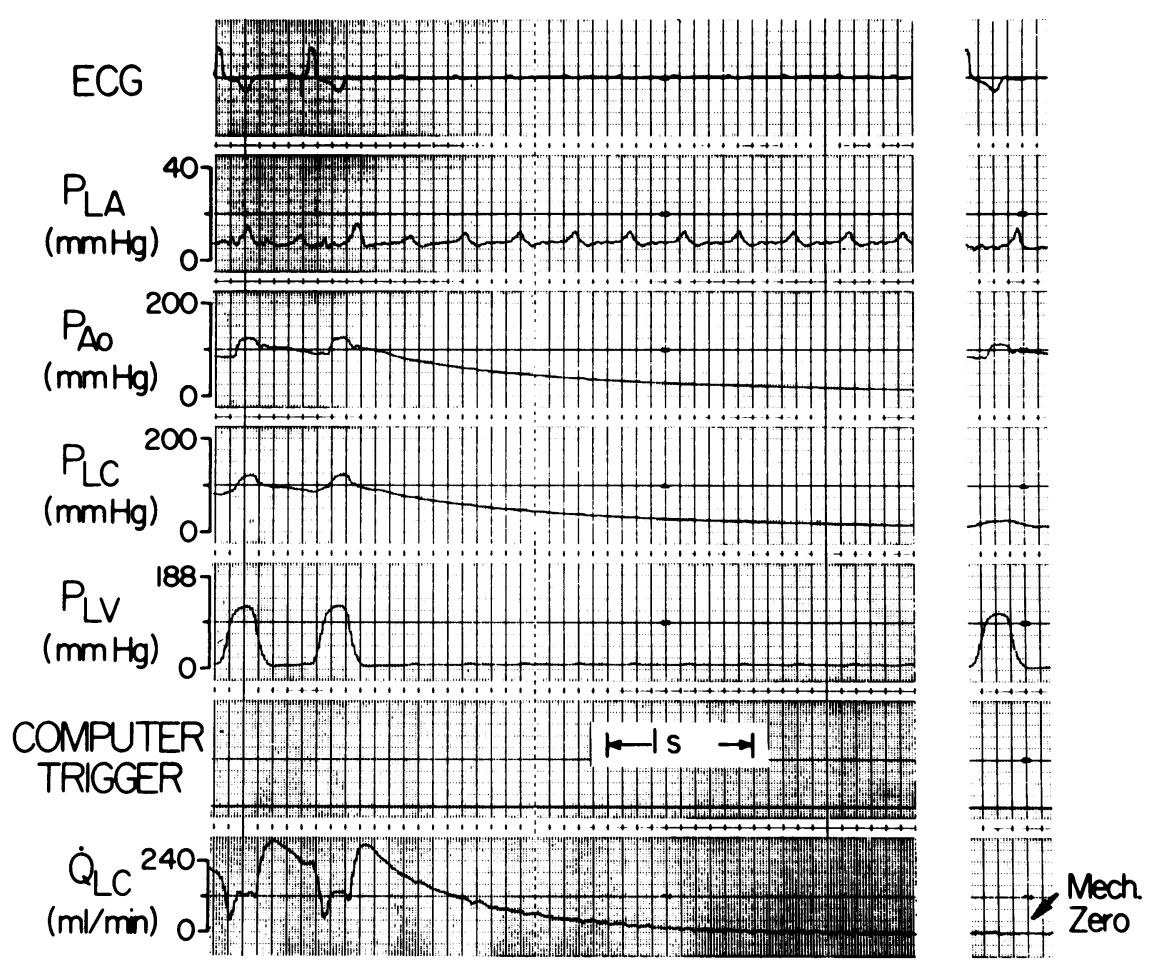

FIGURE 6 Analog record from a prolonged diastole during maximum coronary vasodilation. In this case flow was recorded with an in-line probe. $P_{L A}, P_{A 0}$, $P_{L C}$, and $P_{L V}$, pressures in the left atrium, aorta, left circumflex artery, and left ventricle. $\dot{Q}_{\mathrm{LC}}$, left circumflex inflow. Mech. zero, flowmeter zero recorded during brief mechanical occlusion of the circumflex perfusion circuit. Zero-flow pressure $\left(P_{f=0}\right)$ is $16 \mathrm{~mm} \mathrm{Hg}$.

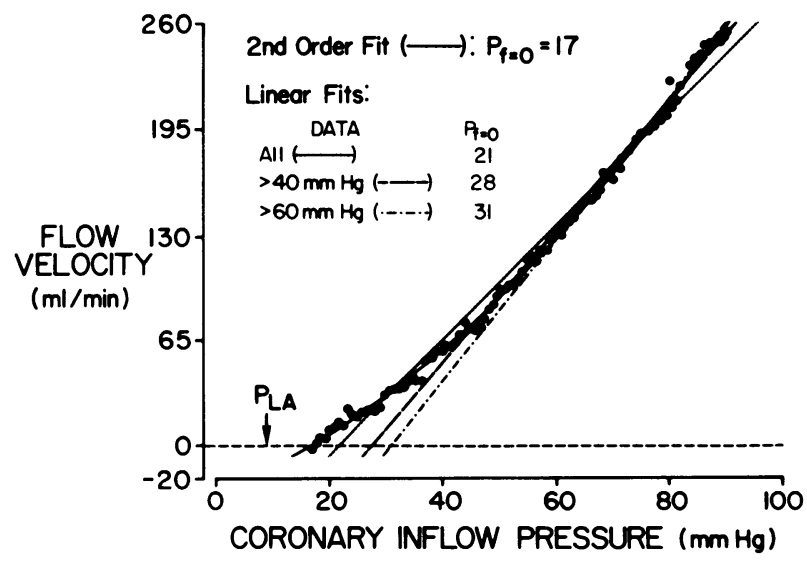

FIGURE 7 Pressure-flow relationship during a single long diastole during maximum vasodilation. Individual data points are shown by solid circles. Despite a correlation coefficient of 0.99 , there is again concavity to the flow axis and data are better fit by a second-order equation than by a linear equation. The second-order zero-flow pressure $\left(P_{f=0}\right), 17 \mathrm{~mm} \mathrm{Hg}$, is distinctly higher than left atrial pressure $\left(\mathrm{P}_{\mathrm{LA}}\right), 9 \mathrm{~mm} \mathrm{Hg}$. The three straight lines represent linear fits for all data points, for data points $>40 \mathrm{~mm} \mathrm{Hg}$, and for data points $>60 \mathrm{~mm} \mathrm{Hg}$. When $P_{f=0}$ is derived by extrapolation in the latter two cases, values substantially exceed those derived from the secondorder fit. cally lower than $P_{f=0}$ during variable-pressure perfusion, the difference averaging $5.9 \pm 3.0 \mathrm{~mm} \mathrm{Hg}$ $(P<0.001)$. Also shown in the Table are data from three animals in which observations were made during sustained maximum vasodilation. There is a small reduction of $P_{f=0}$ during constant-pressure perfusion, averaging $2 \mathrm{~mm} \mathrm{Hg}$. In all studies in Table III, constant-pressure perfusion values of $\mathrm{P}_{\mathrm{f}=0}$ were always higher than simultaneous values of mean left and right atrial pressures $(8 \pm 3$ and $5 \pm 2 \mathrm{~mm} \mathrm{Hg}$, respectively).

\section{DISCUSSION}

The experimental preparation used for these studies was designed so that diastolic pressure-flow relationships could be followed to the point at which coronary inflow actually ceased. Because of the implications of values of $P_{f=0}$ that exceed coronary venous and/or left ventricular diastolic pressures, it was deemed important to determine $P_{f=0}$ without any degree of data extrapolation in both normally reactive and maximally vasodilated coronary beds. A number of technical points relating to the experimental preparation merit comment. The decision to use an acute openchest preparation was taken after preliminary studies 


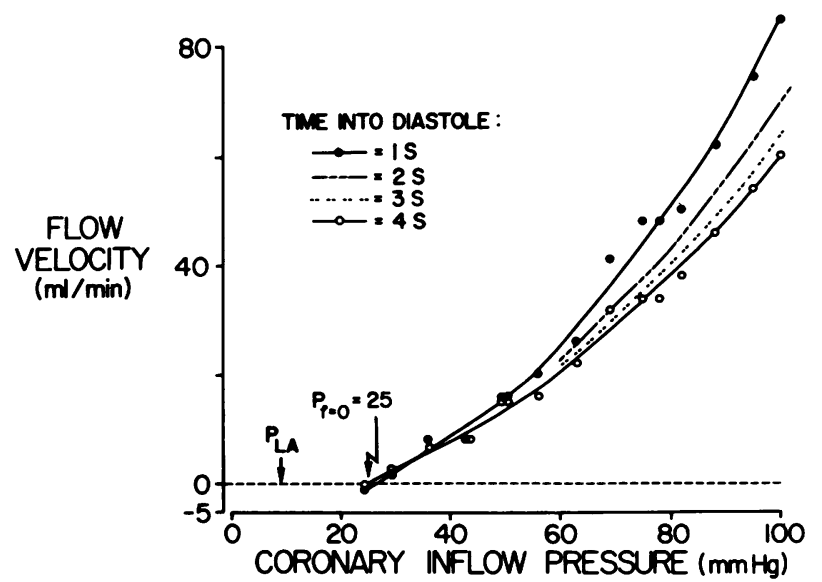

Figure 8 Pressure-flow relationship derived from measurements during 15 long diastoles with constant-pressure reservoir perfusion (before coronary vasodilation). Although circumflex pressure remained constant throughout each diastole, circumflex inflow decreased noticeably with time during diastoles in which inflow pressure was $\geq \sim 60 \mathrm{~mm} \mathrm{Hg}$. The vertical distance between any solid and open circle depicts the change in flow between 1 and $4 \mathrm{~s}$ for that particular level of inflow pressure. Individual data points are not shown for the 2- and 3-s determinations in order not to clutter the figure. Variations of flow with time were appreciably less at lower inflow pressures, and had little effect on the pressureaxis intercept $\left(P_{f=0}\right)$ defined by the series of long diastoles (25 mm Hg).

TABLE III

Second-Order $P_{\mathrm{f}=0}(\mathrm{~mm} \mathrm{Hg})$ in Studies of Capacitive Effects during Long Diastoles*

\begin{tabular}{llc}
\hline \multicolumn{1}{c}{ Dog } & $\begin{array}{c}\text { Variable-pressure } \\
\text { aortic perfusion }\end{array}$ & $\begin{array}{c}\text { Constant-pressure } \\
\text { reservoir perfusion }\end{array}$ \\
\hline $\begin{array}{l}\text { Before vasodilation } \\
14\end{array}$ & $25(n=6)$ & 23 \\
15 & $34(n=7)$ & 30 \\
17 & $24(n=9)$ & 16 \\
18 & $35(n=12)$ & 25 \\
19 & $26(n=5)$ & 23 \\
20 & $25(n=6)$ & 23 \\
21 & $22(n=3)$ & 18 \\
22 & $23(n=6)$ & 15 \\
24 & $28(n=5)$ & 23 \\
25 & $37(n=5)$ & 27 \\
26 & $29(n=7)$ & 20 \\
27 & $24(n=6)$ & 18 \\
Mean & 28 & 4 \\
SD & 5 & 11 \\
During vasodilation & & 11 \\
20 & $12(n=4)$ & 10 \\
$22 \ddagger$ & $15(n=3)$ & 11 \\
$28 \ddagger$ & $12(n=3)$ & \\
Mean & 13 & \\
\hline
\end{tabular}

* Values during variable-pressure aortic perfusion are averages for the number of determinations listed.

t HR, 120 .

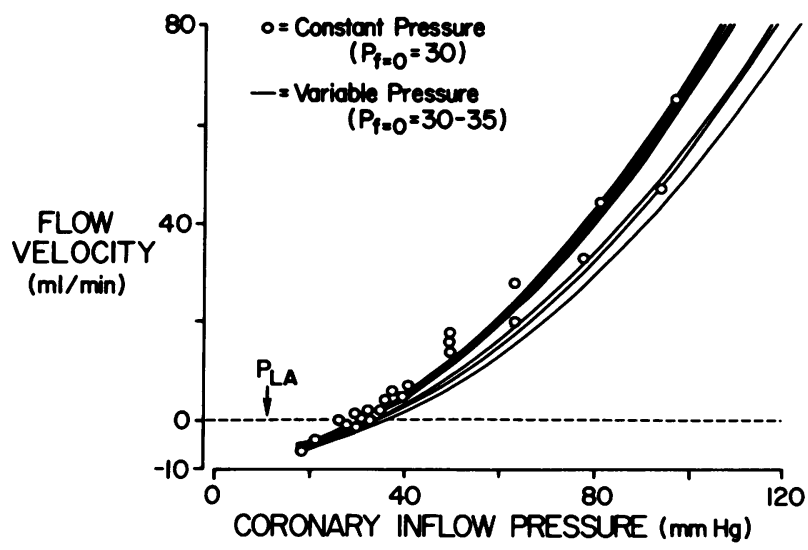

FIGURE 9 Study of capacitive effects in one animal, comparing pressure-flow relationships from variable-pressure aortic perfusion with that derived from constant-pressure reservoir perfusion. The 23 open circles represent values of flow obtained $1 \mathrm{~s}$ after the onset of each of 23 diastoles in which constant-pressure reservoir perfusion ranged from 18 to 97 $\mathrm{mm} \mathrm{Hg}$. The seven solid curves are second-order pressureflow relationships during seven additional long diastoles in which the LC was perfused from the aorta and circumflex pressure decreased in the usual fashion. These 7 determinations were interspersed among the 23 constant-pressure determinations and illustrate the variability of singlediastole pressure-flow relationships during the study. Flows during constant-pressure perfusion are slightly higher than flows at similar inflow pressures during aortic perfusion, but the reduction in zero-flow pressure $\left(P_{f=0}\right)$ for constantvs. variable-perfusion pressure is only $4 \mathrm{~mm} \mathrm{Hg}$.

indicated that $P_{f=0}$ was substantially higher than intracavitary pressures in this preparation as well as in the conscious, chronically instrumented animal. The production of diastoles sufficiently long for inflow to reach zero was facilitated by heart block, since infranodal pacemakers commonly take a few seconds to emerge after cessation of ventricular pacing $(9,10)$. When necessary, emergence of these pacemakers could be delayed further by administration of lidocaine. Aortic venting during the long diastole was an additional useful measure. Preparations were discarded if they showed evidence of progressive coronary dilation, as manifest by spontaneous decreases in $\mathrm{P}_{\mathrm{f}=0}$ and increases in coronary inflow in the face of a stable aortic pressure. The direct measurements of $P_{\mathrm{LC}}$ avoided any effects of differences between aortic and LC pressure contours. Attention was also paid to potential limitations of the electromagnetic measurement of inflow velocity in the acute preparation. Despite the precautions outlined in Methods, acutely applied cuff-type probes remain subject to effects of decreasing vessel diameter as inflow pressure falls during a long diastole. Two lines of evidence suggest that such effects did not importantly affect results in animals in which cuff-type probes were used: $(a)$ values of $P_{f=0}$ and the shape of the pres- 
sure-flow relationship were similar in the $\sim 60 \%$ of animals in which the LC was perfused through an external circuit and inflow was measured by an in-line, cannulating-type flow probe; and $(b)$ simultaneous recordings of LC inflow with cannulating and cuff-type probes in three animals did not differ detectibly.

The present findings support the contention that $P_{f=0}$ is substantially higher than coronary venous outflow pressure and/or left ventricular diastolic pressure, both before and after minimization of vascular smooth muscle tone. The absolute values of $P_{f=0}$ in Table I are somewhat lower than those derived by Bellamy (1) from representative records in conscious dogs with normally reactive coronary beds. They are also lower than his recently reported "basal" values in seven open-chest animals $(49 \pm 6 \mathrm{~mm} \mathrm{Hg})(5)$. At least a portion of these differences may relate to effects of extrapolation in cases in which inflow did not reach zero. Differences in hemodynamics and/or other determinants of $P_{f=0}$ are another important possibility. Our values of $P_{f=0}$ during sustained maximum vasodilation (Table II) are also smaller than those reported by Bellamy $(1,5)$ during reactive hyperemia $(\geq 20 \mathrm{~mm} \mathrm{Hg}$ in conscious dogs and $31 \pm 8 \mathrm{~mm} \mathrm{Hg}$ in open-chest dogs). Effects of extrapolation seem likely to have played a role in these high-flow states; in the present studies, $\mathrm{P}_{\mathrm{f}=0}$ during vasodilation was $\sim 11 \mathrm{~mm} \mathrm{Hg}$ higher when data $<40 \mathrm{~mm} \mathrm{Hg}$ were excluded and $P_{f=0}$ was estimated by linear extrapolation to the pressure axis.

Possible curvilinearity of diastolic pressure-flow relationships was examined more systematically in the present studies than previously $(1,4,5,12)$. This evaluation was facilitated by routinely collecting data to the point of inflow cessation, and by having sufficient data in individual diastoles to avoid the need to use pooled data from several diastoles in constructing pressure-flow relationships. In our preparation, pressure-flow data showed consistent concavity to the flow axis as inflow pressure decreased. Since this curvilinearity was similar before and during maximum coronary vasodilation, it cannot be attributed entirely to changes in vascular smooth muscle tone (6) during the long diastole. It is compatible with a decrease in the number of downstream vascular channels and/or cross sectional area of individual channels as distending pressure falls. Transmural differences in $P_{f=0}$ during maximum vasodilation, with endocardial $P_{f=0}$ exceeding epicardial $P_{f=0}$, have been suggested by Rouleau et al. (2) and our own laboratory (3). Although curvilinearity of pressure-flow relationships is also compatible with increased viscous effects at low flow rates (13), preliminary observations in our laboratory suggest that $P_{f=0}$ is not reduced in the maximally vasodilated bed when hematocrit is lowered acutely by $30-40 \%$. In view of our previous report that $P_{f=0}$ increases in conjunction with increases in preload (3), we have also considered potential effects of increases in ventricular diastolic pressure during long diastoles. During the 217 diastoles included in Tables I and II, left ventricular pressure increased by only $0.9 \pm 0.8 \mathrm{~mm} \mathrm{Hg}$. These increments are small and might be expected to produce convexity rather than concavity to the flow axis.

The substantial decreases in $P_{\mathrm{f}=0}$ associated with vasodilation suggest that vascular smooth muscle tone has an important role in the genesis of $P_{f=0}$ in the normally reactive coronary bed. The correlation of $P_{f=0}$ with inflow pressure in such a bed (Figs. 4 and 5 ) is compatible with the concept that changes in $P_{f=0}$ play a role in adjustments of coronary flow resulting from changes in systemic hemodynamics and other physiological interventions. In the example shown in Fig. 4, $\mathrm{P}_{\mathrm{f}=0}$ decreased without a substantial change in the slope of the pressure-flow relationship, which suggests that diastolic driving pressure was preserved while vascular resistance was unchanged. While this illustration is not intended to minimize the predominance of changes in resistance in physiological adjustments of coronary flow, it suggests that previously unappreciated changes in driving pressure can also be involved. The magnitude of such changes in individual situations requires further evaluation.

The fact that $P_{f=0}$ during maximum vasodilation remained higher than intracavitary diastolic pressures verifies that factors other than vascular smooth muscle tone are also involved in its genesis. These latter factors are probably related to diastolic tissue pressure(s) in the immediate vicinity of the vascular loci of $P_{f=0}$. Measurements of intramyocardial pressure are notoriously difficult (14), with different findings having been reported with different experimental preparations and techniques. An important additional factor is that local stresses in the immediate vicinity of vascular structures need not be reflected in measurements of tissue pressure involving larger areas of the myocardial wall (4). The individual values of $P_{f=0}$ reported in the present studies presumably represent minimum values within a range of $P_{f=0}$ that contribute sufficiently to overall conductance to be detected with the flow-measuring techniques used. Since right atrial pressures were, with one exception, no higher than 6 $\mathrm{mm} \mathrm{Hg}$, the present data do not address Bellamy's recent suggestion that $P_{f=0}$ results from a modified vascular waterfall influenced importantly by coronary venous pressure (5). They do establish that $P_{f=0}$ of substantial magnitude can be associated with quite low values of coronary outflow pressure.

The present studies comparing $P_{f=0}$ during constant- and variable-pressure LC perfusion were intended to address the important possibility raised by Eng et al. (7) that values of $P_{f=0}$ measured during 
single long diastoles are misleadingly high because of capacitive effects within the coronary circulation as inflow pressure decreases. While capacitive discharge from precapillary vessels must cause flow at the capillary level to exceed LC inflow during a long diastole, the important question is the magnitude of this effect. A direct experimental approach seemed advisable because data on in vivo coronary arterial capacitance are limited in both amount and precision. The experimental presumption was that capacitive effects would become minimal during a long diastole in which inflow pressure remained constant. If capacitive effects are normally substantial, the pressure-flow relationship defined by a series of points obtained during constant-pressure perfusion should differ from the relationship obtained during a long diastole in which $P_{L C}$ decreases in the usual fashion. Since the point of particular concern is the value of $P_{f=0}$, attention was focused on flows at lower inflow pressures. As indicated in Table III, values of $P_{f=0}$ obtained during constant-pressure perfusion were slightly but systematically lower than values obtained during conventional variable-pressure perfusion. Differences in individual animals ranged from 2 to 10 and averaged $6 \mathrm{~mm} \mathrm{Hg}$ in the normally reactive vascular bed, and were $2-4 \mathrm{~mm} \mathrm{Hg}$ during maximum vasodilation (when capacitive effects should be less because of substantial increases in flow velocity). Potential effects of collateral circulation in the acute preparation are presumably small but were obviated in three animals by occluding the left anterior descending artery during long diastoles when constant-pressure LC perfusion was used; values of $P_{f=0}$ remained constant within 2-3 $\mathrm{mm} \mathrm{Hg}$. We conclude that capacitive effects during a long diastole do result in higher measured values of $P_{f=0}$. But, the magnitude of this increase is insufficient to negate the conclusion that $P_{f=0}$ is systematically higher than coronary venous outflow pressure and/or left ventricular diastolic pressure in both reactive and dilated coronary beds. Although our conclusions are supported by a study of Kirkeeide et al. (15) in a totally different experimental preparation, they differ from that reported for the nondilated coronary bed by Eng et al. (7), who reported constantpressure perfusion values $P_{f=0}$ of $11-15 \mathrm{~mm} \mathrm{Hg}$ both before and during coronary vasodilation. The reason for their lower values of $P_{f=0}$ before vasodilation is unclear but it may have to do with differences in hemodynamic conditions, the time of flow measurement during long diastoles, or other factors. Capacitive effects could be addressed more directly if technology were available for measuring instantaneous intramyocardial capillary flow during long diastoles.

As illustrated in Fig. 8, flows during long diastoles with constant inflow pressure did vary with time. At pressures $\geqslant 60 \mathrm{~mm} \mathrm{Hg}$, flow decreased substantially throughout the long diastole. The magnitude of timedependent flow reduction was less at lower values of LC perfusion pressure, becoming negligible $<60 \mathrm{~mm}$ $\mathrm{Hg}$. When the experimentally selected constant value of $\mathbf{P}_{\mathrm{LC}}$ was below $\mathbf{P}_{\mathrm{f}=0}$, momentary backflow occurred, presumably as a result of backward capacitive discharge from vessels downstream to the flowmeter. The decreases in flow observed at higher values of $P_{L C}$ are compatible with a vasoconstrictor response to decreased myocardial metabolic demand during a prolonged diastole. The time required for a $50 \%$ decrease in flow averaged $\sim 4 \mathrm{~s}$. While this value could represent the halftime of the response of vascular smooth muscle to changing myocardial $\mathrm{O}_{2}$ requirement, the basis of time-dependent flow responses during long diastoles with constant inflow pressure requires further study. As illustrated in Figs. 8 and 9, these responses do not appear to have influenced the values of $P_{f=0}$ reported in Table III.

As mentioned earlier, the primary goal of the present study was to establish more clearly whether $P_{f=0}$ is higher than traditionally thought. Although observations during prolonged diastoles remain subject to unappreciated limitations of experimental design, we do not presently identify reasons why usual in vivo values of $P_{f=0}$ might be lower than those observed here. Even if circumstances during the latter portion of a long diastole differ from those during a normal length diastole, the initial portion of the pressure-flow relationship during a long diastole is identical to that pertaining during normal diastoles. As illustrated in Fig. 10, extrapolation of pressure-flow data for a normal length diastole to the pressure axis gives values of $P_{f=0}$ that are substantially higher, rather than lower, than those reported here. In addition, resistance calculations in which $P_{f=0}$ is taken as right or left atrial pressure assume a linear pressure-flow relationship that has a distinctly smaller slope than that defined by a linear fit to directly measured values of pressure and flow during the normal length diastole. For atrial pressure to be the correct value of $P_{f=0}$, one must postulate that instantaneous values of inflow during normal diastoles are augmented substantially by capacitive and/or inertial effects. Although further studies addressing this possibility are desirable, data of the type illustrated in Fig. 9 do not indicate systematically lower values of flow with constantpressure perfusion than variable-pressure perfusion at pressures $>60 \mathrm{~mm} \mathrm{Hg}$. We therefore interpret the preponderance of current evidence as indicating that there is a substantial back pressure to flow within the normally functioning coronary bed, and that $P_{f=0}$ is an important determinant of coronary perfusion that has been incompletely appreciated. Fundamental mechanisms underlying $P_{f=0}$ remain to be clarified but probably involve both factors related to, and independent of, 


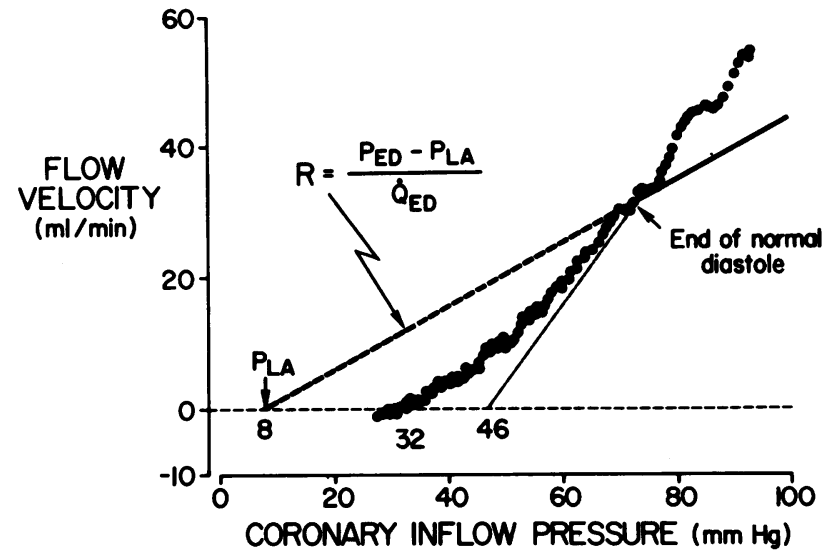

Frgure 10 Comparison of actual values of pressure and flow during the initial portion of a diastole with those expected from a calculation of resistance in which $P_{f=0}$ is taken as atrial pressure. The solid circles again represent data points during a single long diastole (before vasodilation); the second-order $P_{f=0}$ is $32 \mathrm{~mm} \mathrm{Hg}$. The end of a normal length diastole for this animal is indicated by an arrow; if data for the normal diastolic period are linearly extrapolated to the pressure axis, $\mathrm{P}_{\mathrm{f}=0}$ is estimated at $46 \mathrm{~mm} \mathrm{Hg}$. The heavy dashed line depicts the pressure-flow relationship assumed when resistance is calculated at end-diastole, driving pressure being taken as the difference between end-diastolic circumflex pressure $\left(P_{E D}\right)$ and left atrial pressure $\left(P_{L A}\right)$. The actual pressure-flow data for the initial portion of the diastole do not coincide with the solid extension of this traditional resistance line, but instead lie above it. As discussed in the text, this discrepancy is interpreted as further evidence that zero-flow pressures during normal length diastoles are substantially higher than atrial pressures.

vasomotor tone. Adjustments of flow during changing physiological situations may involve changes in $P_{f=0}$ as well as in coronary resistance.

\section{ACKNOWLEDGMENTS}

We gratefully acknowledge the superb technical assistance of our laboratory staff, particularly Charles Soles, Patricia Alsheimer, Richard Kohlmeier, Amy Johnson and Diana Anderson. Dr. Rolf-Eberhard Nitz kindly provided the carbocromen used in these studies.

This study was supported by research grant HLB-15194 from the National Heart, Lung, and Blood Institute, National Institutes of Health.

\section{REFERENCES}

1. Bellamy, R. F. 1978. Diastolic coronary pressure-flow relations in the dog. Circ. Res. 43: 92-101.

2. Rouleau, J., L. E. Boerboom, A. Surjadhảna, and J. I. E. Hoffman. 1979. The role of autoregulation and tissue diastolic pressures in the transmural distribution of left ventricular blood flow in anesthetized dogs. Circ. Res. 45: 804-815.

3. Ellis, A. K., and F. J. Klocke. 1980. Effects of preload on the transmural distribution of perfusion and pressureflow relationships in the canine coronary vascular bed. Circ. Res. 46: 68-77.

4. Bellamy, R. F., and H. S. Lowensohn. 1980. Effect of systole on coronary pressure-flow relations in the right ventricle of the dog. Am. J. Physiol. 238 (Heart Circ. Physiol. 7): H481-H486.

5. Bellamy, R. F., H. S. Lowensohn, W. Ehrlich, and R. W. Baer. 1980. Effect of coronary sinus occlusion on coronary pressure-flow relations. Am. J. Physiol. 239 (Heart Circ. Physiol. 8): H57-H64.

6. Spaan, J. A. E. 1979. Does coronary resistance change only during systole? (Letter to the Editor). Circ. Res. 45: 838-839.

7. Eng, C., J. H. Jentzer, and E. S. Kirk. 1980. Coronary capacitive effects on the high estimates of coronary critical closing pressure. Circulation. 62: III-255. (Abstr.)

8. Steiner, C., and A. T. W. Kovalek. 1968. A simple technique for production of chronic complete heart block in dogs. J. Appl. Physiol. 25: 631-632.

9. Vassalle, M., D. L. Caress, A. J. Slovin, and J. H. Stuckey. 1967. On the cause of ventricular asystole during vagal stimulation. Circ. Res. 20: 228-241.

10. Wittenberg, S. M., P. Gandel, P. M. Hogan, W. Kreuzer, and F. J. Klocke. 1972. Relationship of heart rate to ventricular automaticity in dogs during ouabain administration. Circ. Res. 30: 167-176.

11. Snedecor, G. W., and W. G. Cochran. 1967. Statistical Methods. Iowa State University Press, Ames, Iowa. 453-456.

12. Panerai, R. B., J. H. Chamberlain, and B. McA. Sayers. 1979. Characterization of the extravascular component of coronary resistance by instantaneous pressure-flow relationships in the dog. Circ. Res. 45: 378-390.

13. Dintenfass, L. 1971. Blood Microrheology-Viscosity Factors in Blood Flow, Ischaemia and Thrombosis. Butterworth \& Co. Ltd., London, England. 104-111.

14. Hoffman, J. I. E., and G. D. Buckberg. 1976. Transmural variations in myocardial perfusion. Prog. Cardiol. 5: 37-89.

15. Kirkeeide, R., S. Puschmann, and W. Schaper. 1980. Diastolic coronary pressure-flow relationships investigated by induced long-wave pressure oscillations. Circulation. 62: III-254. (Abstr.) 